\title{
Objective measures of design typicality
}

\section{Stefan Mayer and Jan R. Landwehr}

Goethe University Frankfurt

\begin{abstract}
Author Note
Stefan Mayer, Faculty of Economics and Business Administration, Goethe University Frankfurt, Germany; Jan R. Landwehr, Faculty of Economics and Business Administration, Goethe University Frankfurt, Germany.

Correspondence should be addressed to Stefan Mayer, Goethe University Frankfurt, Chair for Product Management and Marketing Communications, Theodor-W.-Adorno-Platz 4, 60629 Frankfurt am Main/Germany.

E-mail: smayer@wiwi.uni-frankfurt.de
\end{abstract}

Paper published in Design Studies. doi: 10.1016/j.destud.2017.09.004 


\title{
Objective measures of design typicality
}

\begin{abstract}
Design typicality shapes consumers' aesthetic product preferences. Hence, methods for assessing a product design's typicality are vital to enable predictions of a design's market potential. In this research, we introduce four objective measures of design typicality (two based on Euclidian distances between feature points and two based on pixel-wise image correlations) and demonstrate their capability of capturing a subjective typicality experience. Furthermore, we validate the measures in the context of automobile designs with consumer ratings of aesthetic liking and experienced processing fluency by analysing 77 car models from four segments. Our findings identify the most promising approach to quantify design typicality and endorse the use of this measure in future scientific research and in managing product design in business practice.
\end{abstract}

\section{Research Highlights}

- Design typicality significantly predicts aesthetic liking for cars.

- Design typicality can be measured by fully algorithmic measures.

- The experience of processing fluency marginally mediates the effect of typicality.

- Subjective measures of typicality are superior when examining mental processes.

- Objective measures of typicality are recommended for econometric analyses.

Keywords: aesthetics; product design; automotive design; information processing; design typicality 
Visual typicality is a general principle of product design aesthetics, and ample research suggests that consumers prefer prototypical designs over atypical ones (e.g., Graf \& Landwehr, 2017; Hekkert, Snelders, \& van Wieringen, 2003; Veryzer \& Hutchinson, 1998). The link between typicality and aesthetic preferences has been demonstrated in various stimulus domains such as human faces (Langlois \& Roggman, 1990), abstract visual patterns (Martindale \& Moore, 1988; Winkielman, Halberstadt, Fazendeiro, \& Catty, 2006), paintings (Purcell, 1993), and automobile designs (Landwehr, Labroo, \& Herrmann, 2011; Landwehr, Wentzel, \& Herrmann, 2013). Design typicality also plays an important role in consumers' perception of product trendiness (Blijlevens, Mugge, Ye, \& Schoormans, 2013) and in the evaluation of fit between a product and the context of the product's presentation (Blijlevens, Gemser, \& Mugge, 2012).

However, studies showing a link between typicality and aesthetic preferences have been criticized for the possibility that the two constructs are circularly related and mutually influence each other (Boselie, 1991). In particular, whenever participants are asked to subjectively rate a product design's typicality, they may simple rely on their experience of beauty as a heuristic cue to answer the typicality question (Hekkert et al., 2003). Hence, if one is interested in the true typicality of a product design, directly asking people about their subjective typicality perception may be a biased measure because the answer is partly driven by peoples' aesthetic preferences.

Accordingly, the purpose of the present research is to introduce several objective, unbiased measures for design typicality and to compare their ability to predict known consequences of design typicality. In particular, prior research suggests that design typicality facilitates the perceptual processing of the design (i.e., an experience of processing fluency), which is hedonically positive and enhances the aesthetic liking of the design (Reber, Schwarz, \& Winkielman, 2004). Furthermore, objective design typicality should, of course, also be reflected in subjective typicality ratings. In the empirical part of the present paper, we indeed show in the context of automobile designs that some of the proposed measures predict ratings of aesthetic liking, processing fluency, and subjectively perceived typicality by analysing car models from four segments ranging from subcompact cars to SUVs. In doing so, we contribute to research on product design by providing algorithmic, objective approaches to assess design typicality that can be used by scientists and business practitioners alike.

The remainder of the paper is structured as follows. First, we discuss recent theoretical and empirical insights in the fields of design typicality and processing fluency. Next, we 
introduce four objective approaches that measure design typicality and describe their computation. We then present the setup of our empirical study and report the statistical analyses. Finally, we discuss our results, provide ideas for future research, and present scientific and managerial implications.

\section{THEORETICAL BACKGROUND}

When people are forming first impressions, they prefer prototypical stimuli. This "beauty-inaverageness" effect was initially reported for ratings of facial attractiveness (Langlois \& Roggman, 1990). In particular, these authors found that people prefer an average face that is created as a morph of several individual faces (i.e., the visual average of several exemplars) over the individual faces making up the morph. Evolutionary theories explain this preference pattern by the reproductive fitness signalled by typical faces, which are characterized by perfect symmetry and a very smooth skin surface (Rhodes \& Tremewan, 1996). However, subsequent studies demonstrated that this effect also holds true for stimuli that are not associated with a reproductive advantage. Halberstadt and Rhodes (2000), for example, showed that prototypical drawings of dogs and birds and photos of wristwatches were also perceived as more attractive than atypical counterparts. This relationship between typicality and attractiveness is also reflected in ratings of aesthetic liking, defined as "the sensation that results from the perception of attractiveness (or unattractiveness) in products" (Crilly, Moultrie, \& Clarkson, 2004, p. 552; Graf \& Landwehr, 2017). Landwehr and colleagues (2013), for instance, found higher ratings of aesthetic liking for typical car designs when people report their spontaneous liking after a limited amount of exposure to the designs. They further showed that this positive effect of typicality also affects car sales and that the underlying process is processing fluency.

Processing fluency is currently the most influential theoretical explanation for typicality's positive effects on liking and attractiveness (Graf \& Landwehr, 2015; Reber et al., 2004; Winkielman et al., 2006). Fluency refers to the cognitive ease that people experience when perceptually processing a stimulus (Schwarz, 2004). A key prediction of processing fluency theory is that the ease of processing a stimulus determines the aesthetic response towards the stimulus (Reber et al., 2004) because higher fluency and hence easier processing is inherently positive and experienced as gut-level positive affect (Winkielman \& Cacioppo, 2001). 
Importantly, research on fluency has shown that the experience of fluency can be determined by core characteristics of a stimulus that are nonspecific to its content (Reber et al., 2004). People can, for example, process stimuli that are high in symmetry, figure-ground contrast, and visual clarity more efficiently, resulting in feelings of fluency (Reber et al., 2004). Research linking typicality to fluency suggests that prototypical stimuli trigger higher fluency because the traces that are stored in people's visual memory as rudimentary representations of

product categories are activated more quickly and efficiently for typical designs. This has been demonstrated, for example, by Winkielman and colleagues (2006) using random dot patterns. They found that prototypical patterns are classified more efficiently and recruit fewer neural resources.

Against this background, we examine the relationship between design typicality, processing fluency, and aesthetic liking of consumers using digitally rendered car models. In accordance with previous studies on car design (Landwehr et al., 2011, 2013), we argue that consumers will experience greater fluency when processing typical rather than atypical designs and will interpret the fluency signal as their aesthetic liking. We further expect the effect of typicality on aesthetic liking to be mediated by processing fluency.

\section{MEASURING DESIGN TYPICALITY}

In order to capture all possible aspects of typicality, we compare four objective measures of design typicality. Besides their capability to predict ratings of processing fluency and aesthetic liking, we benchmark the four objective measures using their correlations with the traditional way of asking consumers about their subjective typicality impression. In this section, we describe first, the subjective benchmark measure for typicality; second, the two feature-point based objective measure of design typicality; and finally, two objective measures of design typicality based on pixel correlations.

\subsection{Subjective rating of design typicality}

We measured subjective design typicality with a single item successfully employed by prior research in a similar way (Graf \& Landwehr, 2017). In particular, participants were asked in an online survey (see section 3.3) to answer the statement "In comparison to a typical subcompact 
car [compact car/mid-size car/SUV], this car looks ...” on a visual analogue scale (101 points) with the anchors "very atypical" and "very typical".

\subsection{Objective measurement of design typicality}

For all of our objective measures, we consider a prototype as possessing the average values of the visual features of a specific category. This assumption is in line with previous research on prototypicality (Langlois \& Roggman, 1990). Thus, all of our four measures follow the idea that the prototype is a representation of the common characteristics of all designs within a category. A car's design, for example, is typical if the distance to the prototype's design is low, and atypical if the distance to the prototype's design is high. The specific calculation of the prototype and the distance measure, however, differs between the four measures. Two of them are based on Euclidian distances between characteristic feature points of a given design and feature points of an average design (i.e., a morph), the other two are based on image correlations using the pure pixel information of the corresponding digital image.

\section{Feature point-based measures}

One approach that has successfully been used in prior studies on the typicality of car designs (Landwehr et al., 2011, 2013; Liu, Li, Chen, \& Balachander, 2017) is based on feature points. This technique has originally been developed in the field of research on facial attractiveness (Perrett, May, \& Yoshikawa, 1994) and requires that predefined characteristic feature points (e.g., vertex of headlights) are manually set for each image. From that, a morph is created based on the mean position of the feature points. Consequently, the objective typicality measure is created by summing the Euclidean distances between each of the feature points of a particular car and the corresponding feature point of the morph. Since distances from the morph signal atypicality, the score is inverted (i.e., multiplied by -1) to obtain a measure for typicality. We use this approach (subsequently referred to as manually coded feature point measure) as the first of our four objective design typicality measures employing 50 feature points as described in Landwehr et al. (2011).

Our second approach also follows the idea of defining characteristic feature points. However, in contrast to manually coding the feature points, we use an algorithmic approach that is based on perceptual image hashing (Monga \& Evans, 2006). The advantage of this new 
approach is that feature points do not have to be set manually but are found automatically. This makes the analysis of large sets of product designs a simple and quick procedure without the need to manually code thousands of feature points. The procedure consists of three subsequent steps. First, characteristic feature points are identified by the algorithm (e.g., characteristic edges and corners are identified). Second, the distance between the identified set of feature points of any two stimuli is calculated using a Hausdorff-like distance measure (Huttenlocher, Klanderman, \& Rucklidge, 1993). To this end, corresponding feature points between two stimuli are identified via the minimal Euclidean distance between the feature point sets: For instance, for the first feature point of stimulus $A$ the Euclidean distances of this first feature point with the whole set of identified feature points of stimulus $B$ are calculated. The smallest distance reveals the feature point in stimulus $B$ that corresponds most likely to the first feature point in stimulus $A$. This procedure is repeated for all feature points. The sum of the minimal Euclidean distances gives the distance between two images, and repeating the routine for all images produces a matrix of pairwise distances for all stimuli. Third, objective typicality of a given stimulus is calculated by the inverted mean distance per matrix row from all other stimuli, directly following our understanding of a prototype. If a stimulus is very typical for its category, the (mean) visual distance to all other members of the category is low. If the mean visual distance to all other category members is high, then this stimulus is atypical within the category. We call this approach the algorithmically coded feature point measure. The algorithmic detection of the feature points and the calculation of the Euclidean distances is executed in the software package MATLAB.

\section{Image correlation measures}

The third measure is conceptually and computationally very simple and does not rely on characteristic feature points. Hence, it is also applicable to less standardised product shapes (e.g., fashion design). In comparison to the two earlier feature point measures, which require a common base shape in all examined products to make the feature points meaningful, our image correlation measures thus have the beneficial property of being applicable to a much greater number of product categories. For this approach, we first create a mean image based on all images that contains for every pixel the average intensity value across all images. This construes the morphed prototype based on the full image information (think of it as cross-blending all 
images). In a second step, the typicality of a particular image is simply calculated as the correlation of the grayscale values of the image with the grayscale values of the morphed prototype (i.e., the mean image).

We used two versions of the pixel correlation approach. For the first version, we varied the image resolution in steps of $10 \%$ (10 steps from $10 \%$ to $100 \%$ ) to capture all perceptual levels of visual typicality (coarse structures, finer details). Here, typicality is calculated as the average across the typicality values over all resolutions (subsequently referred to as image correlation measure at different resolutions). For the second version, we only use the full pixel resolution. Accordingly, typicality is simply the correlation of the grayscale image with the mean image (subsequently referred to as image correlation measure at full resolution). The calculation of both measures was again done using the software package MATLAB.

\section{STUDY SETUP}

We apply the proposed measures to a database consisting of automobile designs from four segments ranging from subcompact cars to SUVs, and compare them to subjectively rated design typicality. We then estimate several models to predict ratings of aesthetic liking by our measures; and we investigate processing fluency as the psychological mechanism underlying the effect of typicality on aesthetic liking.

\subsection{Stimulus material}

The stimulus material consists of images of digitally rendered greyscale models of cars in a standardised frontal perspective. Overall, 77 cars from 26 brands across 4 segments are used (see Figure 1 for exemplary stimuli). In particular, the stimulus set consists of 17 images of subcompact car models (resolution 306 x 252 pixel), 26 images of compact car models (resolution 294 x 236 pixels), 17 images of mid-size car models (resolution 304 x 235 pixels), and 17 images of SUV models (resolution $305 \times 247$ pixels). The objective measurement of typicality is based on the exact same images (i.e., with respect to resolution and colouring) that we also use for the subjective ratings of liking, fluency, and typicality by a sample of human participants. 

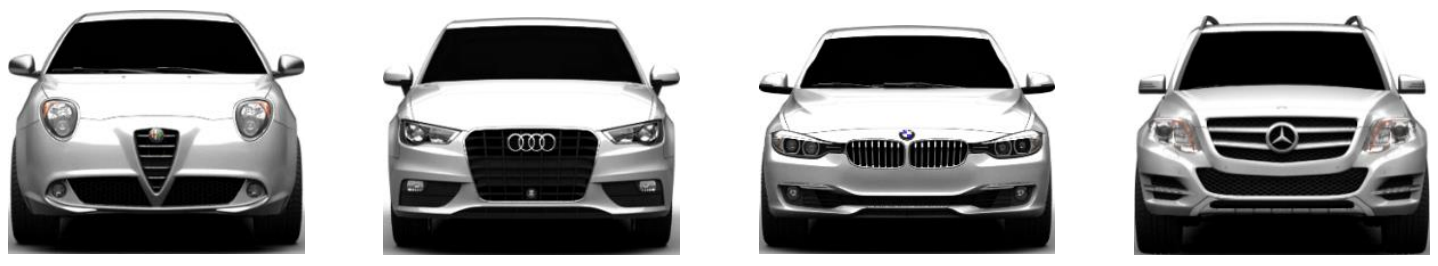

Figure 1 Exemplary car stimuli for each segment (from left to right): subcompact cars, compact cars, mid-size cars, SUVs.

\subsection{Measures for aesthetic liking and fluency}

Aesthetic liking of the car models was measured with one single item ("How much do you like the car's design?", cf. Landwehr et al., 2013). Subjective fluency of the car designs was measured along the easy-difficult dimension ("How easy or difficult is it to study the car's design?", cf. Chang, 2013; Novemsky, Dhar, Schwarz, \& Simonson, 2007). Participants had to rate liking and fluency on a visual analogue scale (101 points from "not at all" to "very much").

\subsection{Participants and design}

To get subjective ratings of aesthetic liking, experienced processing fluency, and perceived typicality, an online survey was conducted in April 2017 using Amazon's MTurk platform. A total of 202 participants completed the survey in one of the four car segments (randomly chosen). Completing the survey took on average approximately 8 minutes and participation was financially compensated. Seven participants were excluded from further analyses due to implausible values ${ }^{1}$. In particular, one participant was excluded after having indicated an age of one; six participants were excluded due to unusually fast or slow completion times (less than three minutes or more than 30 minutes). The resulting final sample therefore consisted of 195 participants $(41.5 \%$ female with an average age of $36.5, \mathrm{SD}=11.64)$. Each participant rated all images within the assigned segment (randomly ordered, one image per page) separately on three dimensions: first on aesthetic liking, second on processing fluency, and finally on subjective typicality. To make the subjectively rated typicality comparable to the objective typicality

\footnotetext{
${ }^{1}$ Excluding the seven participants does not change the pattern of results.
} 
measures, we used the aggregated subjective evaluations ${ }^{2}$ per stimulus for all analyses (i.e., the image-wise means across all evaluations).

\section{RESULTS}

For all analyses, variables were z-standardised per car segment, as the typicality measures were calculated within each segment.

\subsection{Correlation of typicality measures}

Table 1 shows the correlation matrix of all five typicality measures for the 77 car models. All objective typicality measures correlate significantly with the subjectively rated design typicality. The manually coded feature point measure has the highest correlation with the subjective typicality measure $(\mathrm{r}=.501, \mathrm{p}<.001)$. The image correlation measure at different resolutions $(\mathrm{r}$ $=.321, \mathrm{p}=.004)$, the image correlation measure at full resolution $(\mathrm{r}=.312, \mathrm{p}=.006)$ and the algorithmically coded feature point measure $(\mathrm{r}=.307, \mathrm{p}=.007)$ perform almost equally well. Interestingly, both image correlation measures show a higher correlation with the manually coded feature point measure than the algorithmically coded feature point measure.

\footnotetext{
${ }^{2}$ Using the individual-level data yields the same pattern of results.
} 
Table 1 Intercorrelations of the different typicality measures.

\begin{tabular}{|c|c|c|c|c|c|}
\hline Typicality measure & 1 & 2 & 3 & 4 & 5 \\
\hline 1. Subjective & 1 & & & & \\
\hline $\begin{array}{l}\text { 2. Feature points } \\
\text { (manually) }\end{array}$ & $.501 * * *$ & 1 & & & \\
\hline $\begin{array}{l}\text { 3. Feature points } \\
\text { (algorithmically) }\end{array}$ & $.307 * *$ & $.295 * *$ & 1 & & \\
\hline $\begin{array}{l}\text { 4. Image correlation } \\
\text { (different res.) }\end{array}$ & $.321 * *$ & $.599 * * *$ & .100 & 1 & \\
\hline $\begin{array}{l}\text { 5. Image correlation } \\
\text { (only full res.) }\end{array}$ & $.312 * *$ & $.576 * * *$ & .089 & $.995 * * *$ & 1 \\
\hline
\end{tabular}

Notes. $\mathrm{N}=77$ cars. The scores on each measure were standardised per segment prior to analysis. The scores of subjective typicality were obtained by averaging the participants' individual scores per car. Provided are bivariate correlation coefficients. $* * * \mathrm{p}<.001 ; * * \mathrm{p}<.01$

\subsection{Predicting liking and fluency}

To analyse the liking and fluency ratings of the car models, we ran Linear Mixed Models (LMM, Fitzmaurice, Laird, \& Ware, 2004) with REML-fitted estimators and bootstrapped confidence intervals with 1,000 bootstraps. This analysis was conducted on the participant level and, thus, includes a total of 3,764 observations with 17 to 26 nested observations per participant (depending on the number of cars per car segment). Furthermore, as our database includes multiple models from the same car brand, the 77 cars are additionally nested within 26 different brands. The LMM approach takes the nested data structure into account by estimating both fixed and random effects. In particular, because participants evaluated not only one but all car models within a segment, and because there are multiple car models per brand, the error terms of the individual observations cannot be considered independent from each other. Nevertheless, LMM obtain correct estimates when such correlated observations are present. To analyse the data, we relied on the lmer()-function of the $\operatorname{lm} 4$ library of the software R (Bates, Mächler, Bolker, \& Walker, 2015). We ran five separate models (one for each typicality measure) for both liking and 
fluency with a fixed effect for design typicality and a random intercept and a random slope for typicality per participant (to account for individual heterogeneity in participants' preference for typicality) and an additional random intercept per car brand. Thus, the models we used to predict liking and fluency of a car $h$ nested within participant $i$ and additionally also nested within car brand $j$ had the following form, where $b$ indicates the fixed effects (standardised beta coefficients), $u$ indicates the random effects, and $e_{h i j}$ indicates the residual:

$$
\begin{aligned}
& \text { LIKING }_{h i j}=b_{0}+b_{1} \times \text { TYPICALITY }_{h j}+u_{0 i}+u_{0 j}+u_{1 i} \times \text { TYPICALITY }_{h j}+e_{h i j} \\
& \text { FLUENCY }_{h i j}=b_{0}+b_{1} \times \text { TYPICALITY }_{h j}+u_{0 i}+u_{0 j}+u_{1 i} \times \text { TYPICALITY }_{h j}+e_{h i j}
\end{aligned}
$$

Table 2 shows the results of all five models for aesthetic liking as the dependent variable. For all estimated models the $95 \%$ confidence intervals indicate a significant and positive effect of the corresponding typicality measure except for the algorithmic feature point measure (95\% CI includes zero, cf. Table 2). In particular, the strongest effect can be found for the subjective typicality measure $(b=.129, t=4.63,95 \%$ CI $[.076, .176])$, followed by the manually coded feature point measure $(b=.064, t=2.80,95 \% \mathrm{CI}[.021 ; .102])$, and both variants of the image correlation measure (image correlation measure at different resolutions: $b=.036, t=2.08,95 \%$ CI [.002; .069]; image correlation measure at full resolution: $b=.036, t=2.05,95 \% \mathrm{CI}[.002$; $.069])$. 
Table 2 Comparison of the different LMM models predicting aesthetic liking (baseline model).

\begin{tabular}{lccccc}
\hline Typicality measure & Estimate & $95 \% \mathrm{CI}$ & Std. Err. & $\mathrm{t}$-Value & $\mathrm{R}^{2}$ \\
\hline Subjective & .129 & {$[.076, .176]$} & .028 & 4.63 & .402 \\
& & & & & \\
$\begin{array}{l}\text { Feature points } \\
\text { (manually) }\end{array}$ & .064 & {$[.021, .102]$} & .023 & 2.80 & .391 \\
$\begin{array}{l}\text { Feature points } \\
\text { (algorithmically) }\end{array}$ & .005 & {$[-.035, .046]$} & .023 & 0.22 & .380 \\
$\begin{array}{l}\text { Image correlation } \\
\text { (different res.) }\end{array}$ & .036 & {$[.002, .069]$} & .017 & 2.08 & .358 \\
$\begin{array}{l}\text { Image correlation } \\
\text { (only full res.) }\end{array}$ & .036 & {$[.002, .069]$} & .018 & 2.05 & .358 \\
\end{tabular}

Notes. Data basis are 3,764 observations of $\mathrm{N}=195$ participants for each analysis. The cars' scores on each typicality measure were standardised per segment prior to analysis. The scores of subjective typicality were obtained by averaging the participants' individual scores per car. All models were fitted by REML and include a random intercept and a random slope for typicality per participant as well as a random intercept per car brand. $\mathrm{R}^{2}$ is the squared multiple correlation between the fitted and the observed values of aesthetic liking. The $95 \%$ confidence intervals are based on 1,000 bootstrap samples.

The subjective fluency experience, on the other hand, is only associated with the subjective typicality measure ( $b=.095, t=4.31,95 \%$ CI $[.053 ; .134]$; cf. Table 3$)$. The manually coded feature point measure as well as the remaining objective typicality measures are not systematically related to processing fluency; all their $95 \%$ confidence intervals (see Table 3 ) and $90 \%$ confidence intervals include zero. 
Table 3 Comparison of the different LMM models predicting processing fluency (mediator model).

\begin{tabular}{lccccc}
\hline Typicality measure & Estimate & $95 \% \mathrm{CI}$ & Std. Err. & $\mathrm{t}$-Value & $\mathrm{R}^{2}$ \\
\hline Subjective & .095 & {$[.053, .134]$} & .022 & 4.31 & .542 \\
& & & & & \\
$\begin{array}{l}\text { Feature points } \\
\text { (manually) }\end{array}$ & .025 & {$[-.009, .058]$} & .019 & 1.35 & .511 \\
$\begin{array}{l}\text { Feature points } \\
\text { (algorithmically) }\end{array}$ & -.003 & {$[-.036, .026]$} & .018 & -0.18 & .486 \\
$\begin{array}{l}\text { Image correlation } \\
\text { (different res.) }\end{array}$ & .016 & {$[-.018, .048]$} & .016 & 1.00 & .496 \\
$\begin{array}{l}\text { Image correlation } \\
\text { (only full res.) }\end{array}$ & .016 & {$[-.018, .047]$} & .016 & 0.98 & .497 \\
\end{tabular}

Notes. Data basis are 3,764 observations of $\mathrm{N}=195$ participants for each analysis. The cars' scores on each typicality measure were standardised per segment prior to analysis. The scores of subjective typicality were obtained by averaging the participants' individual scores per car. All models were fitted by REML and include a random intercept and a random slope for typicality per participant as well as a random intercept per car brand $(\mathrm{N}=26) . \mathrm{R}^{2}$ is the squared multiple correlation between the fitted and the observed values of aesthetic liking. The $95 \%$ confidence intervals are based on 1,000 bootstrap samples.

\subsection{Mediation Analysis}

To investigate whether processing fluency mediates the effect of typicality on aesthetic liking, we ran mediation analyses according to MacKinnon (2008). To this end, we estimated for each typicality measure in addition to the baseline model (described in equation 1) and the mediator model (described in equation 2) a full model where we regressed aesthetic liking on both typicality and processing fluency: 


$$
\begin{aligned}
\text { LIKING }_{h i j}= & b_{0}+b_{1} \times \text { TYPICALITY }_{h j}+b_{2} \times \text { FLUENCY }_{h i j}+u_{0 i}+u_{0 j}+u_{1 i} \times \text { TYPICALITY }_{h j} \\
& +e_{h i j}
\end{aligned}
$$

The results are given in Table 4. In comparison to equation (1), including processing fluency as an additional predictor of aesthetic liking as shown in equation (3) decreased the effect of typicality for the subjective typicality measure from $b=.129$ (cf. Table 2) to $b=.117$ ( $t$ $=4.22,95 \%$ CI $[.062, .164]$; cf. Table 4$)$, which suggests that processing fluency partially mediates the effect of subjective typicality. An additionally computed bootstrapped $95 \%$ confidence interval for the corresponding indirect effect (Hayes \& Rockwood, 2016) corroborates the significance of this finding $\left(b_{\text {indirect }}=.013,95 \%\right.$ CI $\left.[.006, .021]\right)$. For the remaining objective measures, both $90 \%$ and $95 \%$ confidence intervals of the indirect effect include zero, suggesting that their effect on aesthetic liking is not mediated by processing fluency.

Figure 2 summarizes the results of all models described in section 4.2 and 4.3. 
Table 4 Comparison of the different LMM models predicting aesthetic liking by measured typicality and processing fluency (full model).

\begin{tabular}{|c|c|c|c|c|c|c|c|c|c|}
\hline \multirow[b]{2}{*}{ Typicality measure } & \multicolumn{4}{|c|}{ Typicality } & \multicolumn{4}{|c|}{ Fluency } & \multirow[b]{2}{*}{$\mathrm{R}^{2}$} \\
\hline & Estimate & $95 \% \mathrm{CI}$ & Std. Err. & $\mathrm{t}-$ Value & Estimate & $95 \% \mathrm{CI}$ & Std. Err. & $\mathrm{t}-$ Value & \\
\hline Subjective & .117 & {$[.062, .164]$} & .028 & 4.22 & .1312 & {$[.084, .178]$} & .0178 & 7.37 & .410 \\
\hline $\begin{array}{l}\text { Feature points } \\
\text { (manually) }\end{array}$ & .061 & {$[.019, .099]$} & .023 & 2.70 & .1329 & {$[.087, .179]$} & .0177 & 7.52 & .400 \\
\hline $\begin{array}{l}\text { Feature points } \\
\text { (algorithmically) }\end{array}$ & .007 & {$[-.033, .047]$} & .023 & 0.28 & .1331 & {$[.088, .180]$} & .0176 & 7.55 & .388 \\
\hline $\begin{array}{l}\text { Image correlation } \\
\text { (different res.) }\end{array}$ & .034 & {$[.001, .066]$} & .017 & 1.95 & .1361 & {$[.089, .184]$} & .0177 & 7.69 & .369 \\
\hline $\begin{array}{l}\text { Image correlation } \\
\text { (only full res.) }\end{array}$ & .034 & {$[.-000, .067]$} & .018 & 1.92 & .1361 & {$[.089, .184]$} & .0177 & 7.69 & .369 \\
\hline
\end{tabular}

Notes. Data basis are 3,764 observations of $\mathrm{N}=195$ participants for each analysis. The cars' scores on each typicality measure were standardised per segment prior to analysis. The scores of subjective typicality were obtained by averaging the participants' individual scores per car. All models were fitted by REML and include a random intercept and a random slope for typicality per participant as well as a random intercept per car brand. $\mathrm{R}^{2}$ is the squared multiple correlation between the fitted and the observed values of aesthetic liking. The $95 \%$ confidence intervals are based on 1,000 bootstrap samples. 


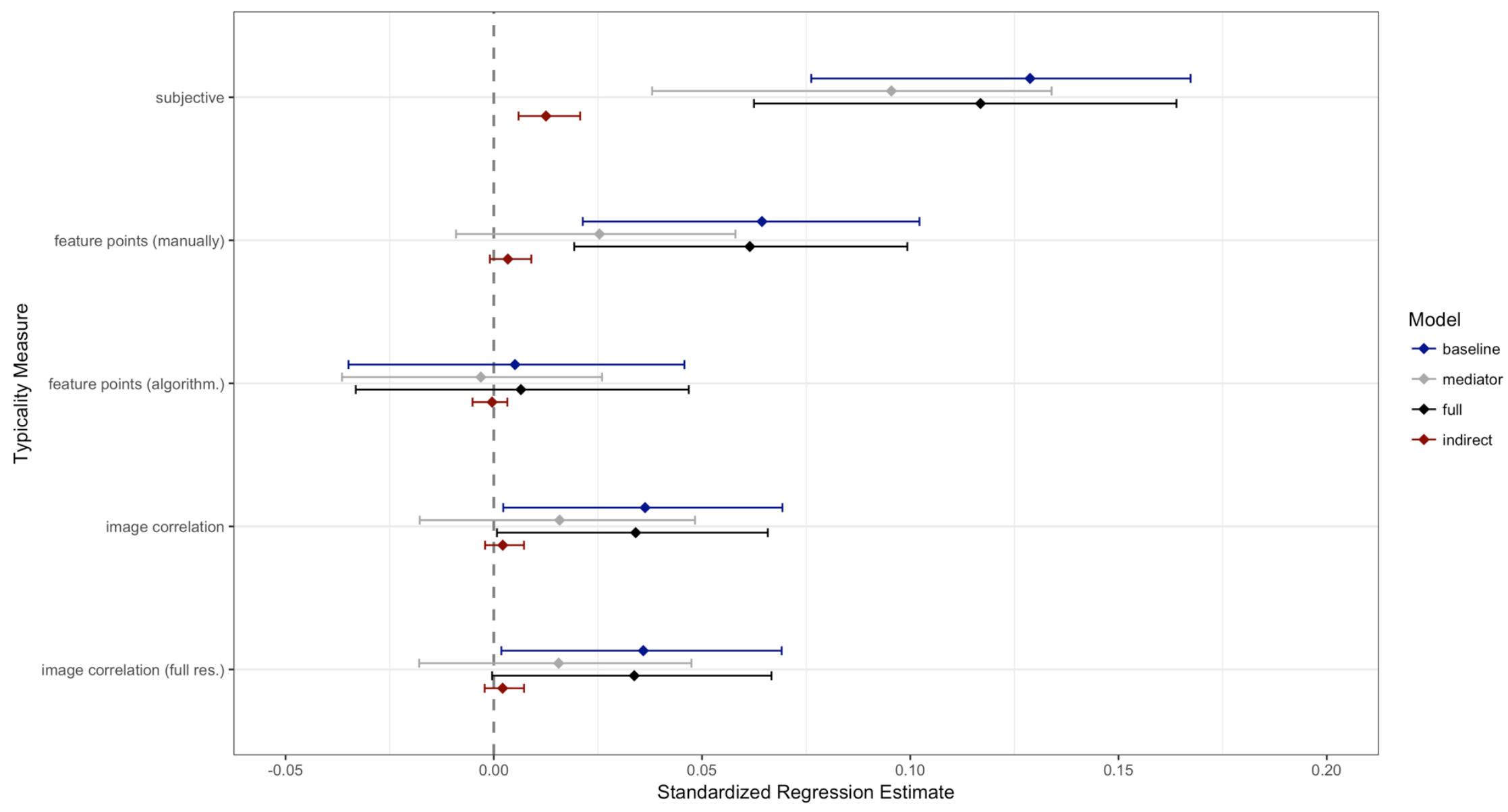

Figure 2 Results of all Linear Mixed Models (LMMs) as a function of the employed typicality measure. In the baseline model, aesthetic liking is regressed on the corresponding typicality measure. The mediator model predicts processing fluency by the typicality measures. The full model predicts liking by typicality and fluency (the results for fluency in the full model are not shown in the figure). The indirect effect of typicality on aesthetic liking via processing fluency is also depicted. Data basis for all models are 3,764 observations of $\mathrm{N}=195$ participants for each model. All models were fitted by REML 
and include a random intercept and a random slope for typicality per participant as well as a random intercept per car brand. Shown are the point-estimates from the LMMs with bootstrapped $95 \%$ confidence intervals. 


\section{DISCUSSION}

Design typicality substantially influences consumers' aesthetic evaluations of products. Hence, reliably assessing a product design's typicality plays a key role in predicting consumers' responses to a design. However, directly asking people for their subjective typicality experience may yield a biased measure as the rating arguably contains the overall aesthetic impression of the product. Although this can be useful for predictions, this approach may not be used as an unbiased measure of a design's typicality. Therefore, the aim of the present research was to examine whether unbiased objective measures of design typicality can capture a subjective typicality experience and predict outcomes of typicality. In doing so, we are the first to provide several objective measures of design typicality, and to systematically compare them with subjective typicality. Furthermore, we provide novel evidence for their predictive strength regarding processing fluency and aesthetic liking.

In our empirical analysis of car designs, we first looked at the correlations of the proposed objective measures with the subjectively reported typicality experience. Results show that all four proposed objective typicality measures substantially correlate with the subjective measure. We further estimated a series of Linear Mixed Models predicting aesthetic liking and processing fluency with each of the four objective measures and the subjective measure. We find that the manually coded feature point measure significantly predicts aesthetic liking but not processing fluency. Both image correlation based measures perform equally well in significantly predicting aesthetic liking. Additional analyses show that they perform very similarly because of the robustness of the approach to changes in image resolutions (see Figures A1 and A2 in the Appendix). However, they are not systematically related to processing fluency. Although our algorithmically coded feature point measure is correlated with the subjective perception of typicality, it is not related to aesthetic liking and fluency. Hence, we would not recommend this approach for future applications. In contrast, subjectively rated typicality has in all models the strongest effect on liking and fluency. Yet, as the database only contained 77 car models, the objective measures could have performed better if the study design had more power.

The positive effects of typicality revealed are in line with established theory and prior research (Landwehr et al., 2011; Reber et al., 2004). Moreover, processing fluency seems to 
contribute to the positive effect of typicality on aesthetic liking. If comparing the subjective with the objective design typicality measures, we find that the subjective measure is best suited to uncovering such an underlying psychological process. This fact, however, is not surprising, as the subjective measure may be biased towards the overall aesthetic impression so that it is highly linked to fluency and liking. Hence, a recommendation would be to use the subjective typicality measure if one is interested in the psychological process. However, if one's goal is to econometrically model the effect of (pure) typicality, it seems also adequate to use the manual feature point measure, thereby avoiding the reversed causality problem. Moreover, in order to study the effect of typicality on aesthetic liking using large datasets, the image correlation measure may be most advisable, as it is likewise significantly related to liking and can be fully automated.

In terms of future research, our study suggests going further into the different aspects of design typicality that can be captured by objective measures. While our feature point measures focus on salient product characteristics, the correlation based measures incorporate every visual aspect present in a design. It would be interesting to examine whether these different approaches also capture different aspects in people's subjective perception/experience. With respect to the correlation based measures, it would also be interesting to theoretically understand why the predictive strength of the measures is robust against different image resolutions. This finding suggests that typicality is a holistic impression that does not depend on the information present in the finer details of the design. A final question that could be addressed by future research is concerned with the psychological processes that are responsible for the link between typicality and aesthetic liking. Our analyses showed that processing fluency may act as a mediator but that its effect is rather small and a large portion of the variance in aesthetic liking seems to be triggered by different processes. Future research should examine whether there are other mediators that need to be considered or whether our measures could be improved to better capture psychological processes.

Overall, our findings demonstrate the possibility of using algorithmic, objective measures of design typicality to predict consumers' aesthetic preferences. Our research thereby contributes to the design community by providing a systematic comparison of four such measures with respect to their strengths and weaknesses. Our measures are simple to implement and can be used by researchers in the area of empirical aesthetics and product design as well as by practitioners 
interested in evaluating the potential success of new product designs. In sum, our findings endorse the general notion that objective measures should be included in product design research due to their robust capability of quantifying aesthetics. 


\section{REFERENCES}

Bates, D., Mächler, M., Bolker, B., \& Walker, S. (2015). Fitting Linear Mixed-Effects Models Using lme4. Journal of Statistical Software, 67(1), 1-48. http://doi.org/10.18637/jss.v067.i01

Blijlevens, J., Gemser, G., \& Mugge, R. (2012). The importance of being “well-placed": The influence of context on perceived typicality and esthetic appraisal of product appearance. Acta Psychologica, 139(1), 178-86. http://doi.org/10.1016/j.actpsy.2011.11.004

Blijlevens, J., Mugge, R., Ye, P., \& Schoormans, J. P. L. (2013). The influence of product exposure on trendiness and aesthetic appraisal. International Journal of Design, 7(1), 5567.

Boselie, F. (1991). Against Prototypicality as a Central Concept in Aesthetics. Empirical Studies of the Arts, 9(1), 65-73. http://doi.org/10.2190/ERDR-FN28-PUEE-EU7F

Chang, C. (2013). Imagery Fluency and Narrative Advertising Effects. Journal of Advertising, 42(1), 54-68. http://doi.org/10.1080/00913367.2012.749087

Crilly, N., Moultrie, J., \& Clarkson, P. J. (2004). Seeing Things: Consumer Response to the Visual Domain in Product Design. Design Studies, 25(6), 547-577. http://doi.org/10.1016/j.destud.2004.03.001

Fitzmaurice, G. M., Laird, N. M., \& Ware, J. H. (2004). Applied Longitudinal Analysis. Hoboken, NJ: John Wiley \& Sons.

Graf, L. K. M., \& Landwehr, J. R. (2015). A Dual-Process Perspective on Fluency-Based Aesthetics: The Pleasure-Interest Model of Aesthetic Liking. Personality and Social Psychology Review, 19(4), 395-410. http://doi.org/10.1177/1088868315574978

Graf, L. K. M., \& Landwehr, J. R. (2017). Aesthetic Pleasure versus Aesthetic Interest: The Two Routes to Aesthetic Liking. Frontiers in Psychology, 8(15). http://doi.org/10.3389/fpsyg.2017.00015

Halberstadt, J., \& Rhodes, G. (2000). The Attractiveness of Nonface Averages: Implications for an Evolutionary Explanation of the Attractiveness of Average Faces. Psychological Science, 11(4), 285-289. http://doi.org/10.1111/1467-9280.00257 
Hayes, A. F., \& Rockwood, N. J. (2016). Regression-based statistical mediation and moderation analysis in clinical research: Observations, recommendations, and implementation. Behaviour Research and Therapy, in press. http://doi.org/10.1016/j.brat.2016.11.001

Hekkert, P., Snelders, D., \& van Wieringen, P. C. W. (2003). "Most Advanced, Yet Acceptable": Typicality and Novelty as Joint Predictors of Aesthetic Preference in Industrial Design. British Journal of Psychology, 94(1), 111-124. http://doi.org/10.1348/000712603762842147

Huttenlocher, D. P., Klanderman, G. A., \& Rucklidge, W. J. (1993). Comparing images using the Hausdorff distance. IEEE Transactions on Pattern Analysis and Machine Intelligence, 15(9), 850-863. http://doi.org/10.1109/34.232073

Landwehr, J. R., Labroo, A. A., \& Herrmann, A. (2011). Gut Liking for the Ordinary: Incorporating Design Fluency Improves Automobile Sales Forecasts. Marketing Science, 30(3), 416-429. http://doi.org/10.1287/mksc.1110.0633

Landwehr, J. R., Wentzel, D., \& Herrmann, A. (2013). Product Design for the Long Run: Consumer Responses to Typical and Atypical Designs at Different Stages of Exposure. Journal of Marketing, 77(5), 92-107. http://doi.org/10.1509/jm.11.0286

Langlois, J. H., \& Roggman, L. A. (1990). Attractive Faces Are Only Average. Psychological Science, 1(2), 115-121. http://doi.org/10.1111/j.1467-9280.1990.tb00079.x

Liu, Y., Li, K. J., Chen, H. (Allan), \& Balachander, S. (2017). The Effects of Products' Aesthetic Design on Demand and Marketing-Mix Effectiveness: The Role of Segment Prototypicality and Brand Consistency. Journal of Marketing, 81(1), 83-102. http://doi.org/10.1509/jm.15.0315

MacKinnon, D. P. (2008). Introduction to Statistical Mediation Analysis. New York, NY: Erlbaum and Taylor \& Francis Group. http://doi.org/10.1037/13620-018 Martindale, C., \& Moore, K. (1988). Priming, prototypicality, and preference. Journal of Experimental Psychology: Human Perception and Performance, 14(4), 661-670. http://doi.org/10.1037//0096-1523.14.4.661

Monga, V., \& Evans, B. L. (2006). Perceptual Image Hashing Via Feature Points: Performance Evaluation and Trade-Offs. IEEE Transactions on Image Processing, 15(11), 3452-3465. http://doi.org/10.1109/TIP.2006.881948

Novemsky, N., Dhar, R., Schwarz, N., \& Simonson, I. (2007). Preference Fluency in Choice. 
Journal of Marketing Research, 44(3), 347-356. http://doi.org/10.1509/jmkr.44.3.347

Perrett, D. I., May, K. A., \& Yoshikawa, S. (1994). Facial shape and judgements of female attractiveness. Nature, 368(6468), 239-242. http://doi.org/10.1038/368239a0

Purcell, A. T. (1993). Relations between Preference and Typicality in the Experience of Paintings. Leonardo, 26(3), 235-241. http://doi.org/10.2307/1575817

Reber, R., Schwarz, N., \& Winkielman, P. (2004). Processing Fluency and Aesthetic Pleasure: Is Beauty in the Perceiver's Processing Experience? Personality and Social Pschology Review, 8(4), 364-382. http://doi.org/10.1207/s15327957pspr0804_3

Rhodes, G., \& Tremewan, T. (1996). Averageness, exaggeration, and facial attractiveness. Psychological Science, 7(2), 105-110. http://doi.org/10.1111/j.1467-9280.1996.tb00338.x

Schwarz, N. (2004). Metacognitive Experiences in Consumer Judgment and Decision Making. Journal of Consumer Psychology, 14(4), 332-348. http://doi.org/10.1207/s15327663jcp1404_2

Veryzer, R. W., \& Hutchinson, J. W. (1998). The Influence of Unity and Prototypicality on Aesthetic Responses to New Product Designs. Journal of Consumer Research, 24(4), 374385. http://doi.org/10.1086/209516

Winkielman, P., \& Cacioppo, J. T. (2001). Mind at Ease Puts a Smile on the Face: Psychophysiological Evidence That Processing Facilitation Elicits Positive Affect. Journal of Personality and Social Psychology, 81(6), 989-1000. http://doi.org/10.1037//00223514.81.6.989

Winkielman, P., Halberstadt, J., Fazendeiro, T., \& Catty, S. (2006). Prototypes are attractive because they are easy on the mind. Psychological Science, 17(9), 799-806. http://doi.org/10.1111/j.1467-9280.2006.01785.x 


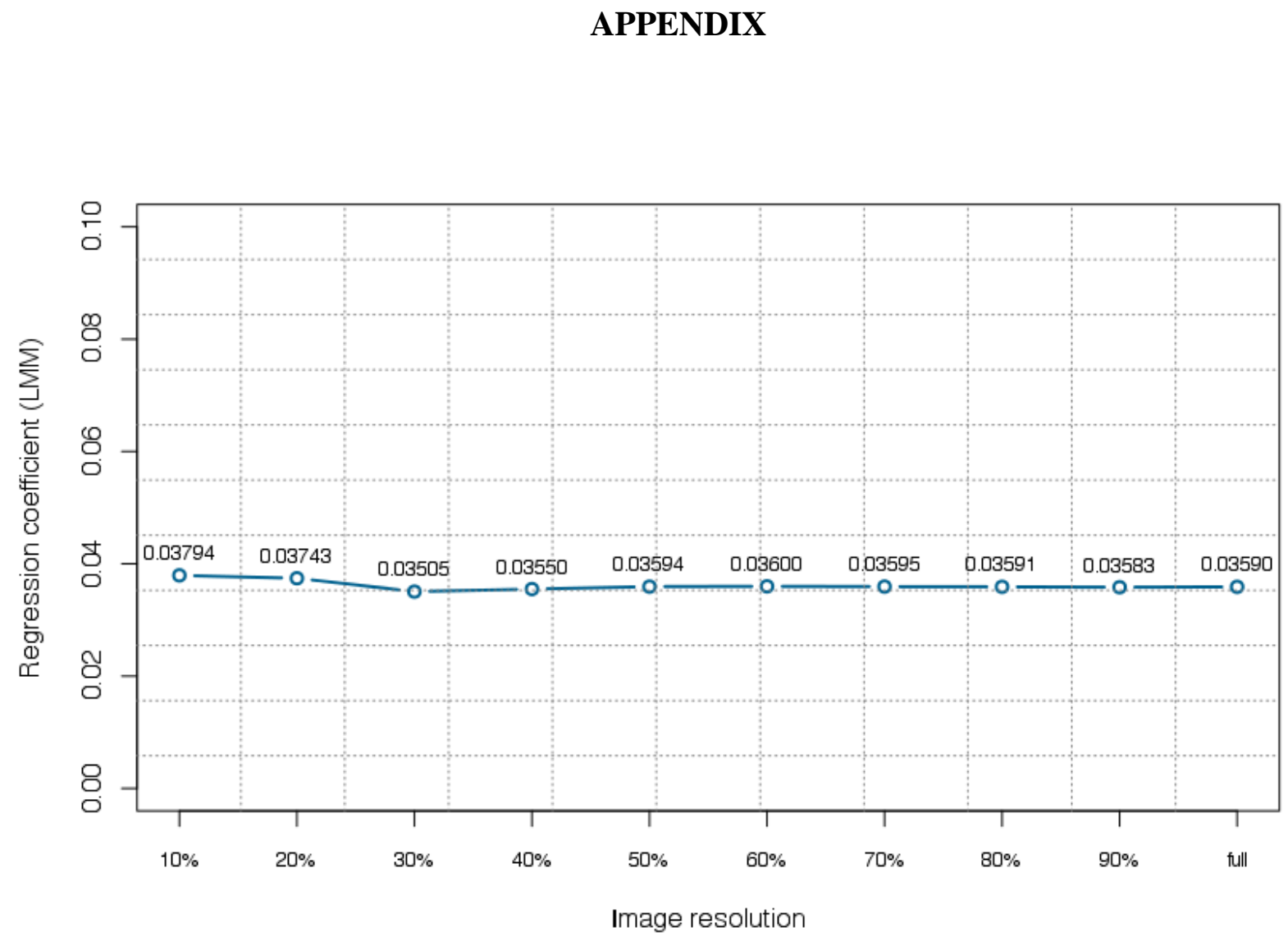

Figure A1 Effect of typicality (measured with the image correlation measure) on aesthetic liking as a function of image resolution. 


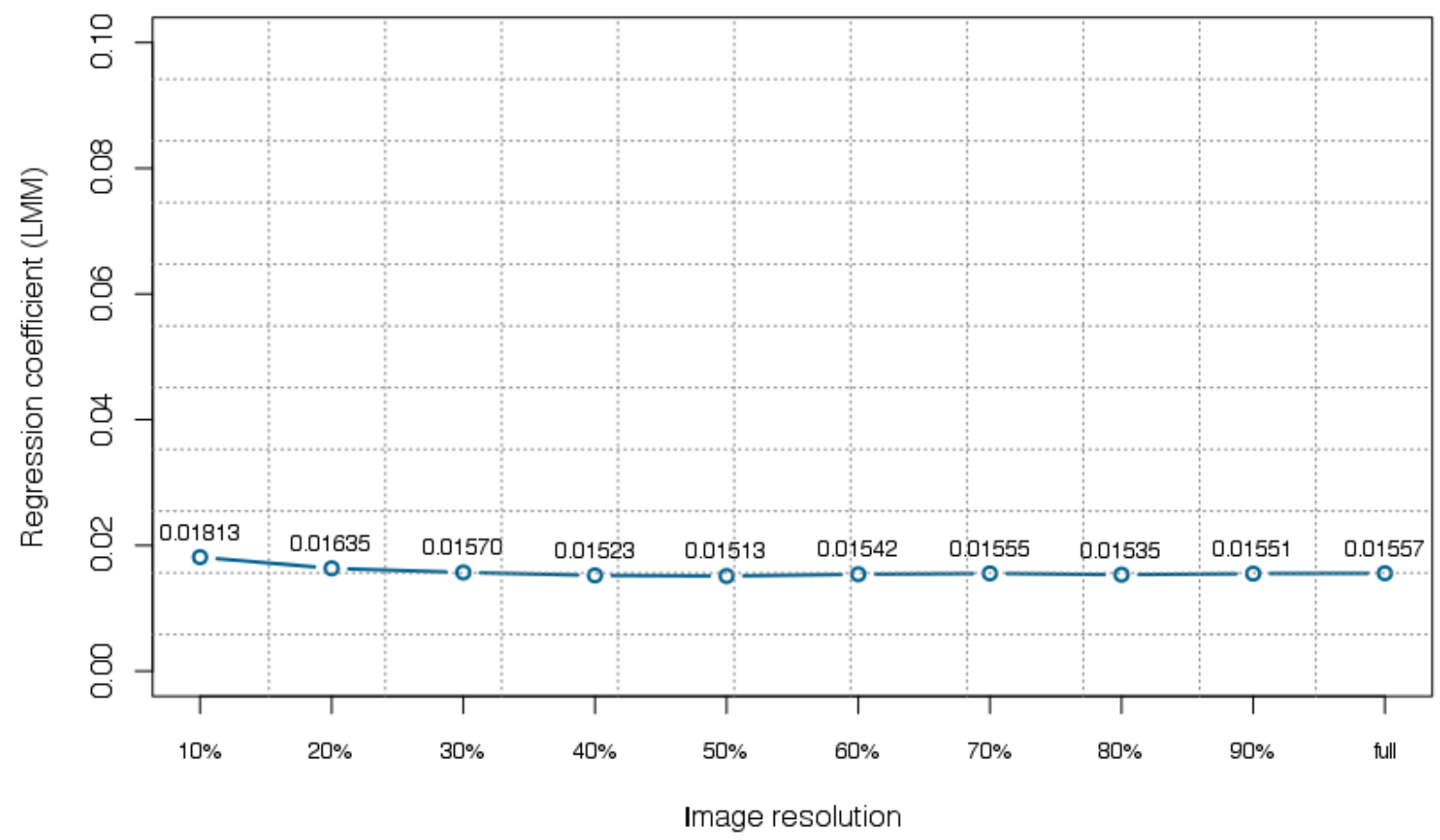

Figure A2 Effect of typicality (measured with the image correlation measure) on processing fluency as a function of image resolution. 